\title{
Sacrifice et transfert des âmes chez les Songhay du Niger
}

Sacrifice and the transfer of souls among the Songhai in Niger

Jean Rouch

\section{(2) OpenEdition}

\section{Journals}

Édition électronique

URL : http://journals.openedition.org/span/298

DOI : $10.4000 /$ span.298

ISSN : 2268-1558

Éditeur

École pratique des hautes études. Sciences humaines

\section{Édition imprimée}

Date de publication : 1 septembre 1976

Pagination : 55-66

ISSN : 0294-7080

\section{Référence électronique}

Jean Rouch, «Sacrifice et transfert des âmes chez les Songhay du Niger », Systèmes de pensée en Afrique noire [En ligne], 2 | 1976, mis en ligne le 04 juin 2013, consulté le 23 avril 2019. URL : http:// journals.openedition.org/span/298; DOI : 10.4000/span.298 


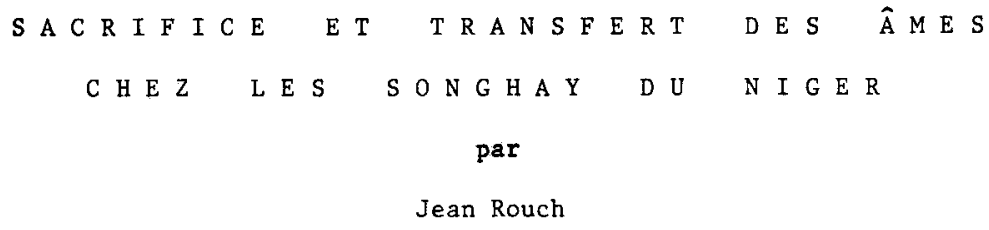

Dans "Remarques sur le mécanisme du sacrifice dogon (Soudan français)" (Journal de la Société des Africanistes, X, 1940, 127-130), Marce1 Griaule écrit :

"le sacrifice est l'acte central de nombreux rites pratiquês dans le culte des morts ou dans le service ordinaire cies puissances, à des fins de purification, de consécration, de divination, de propitiation entre autres."

Dans le sacrifice, Griaule part de ce schéma :

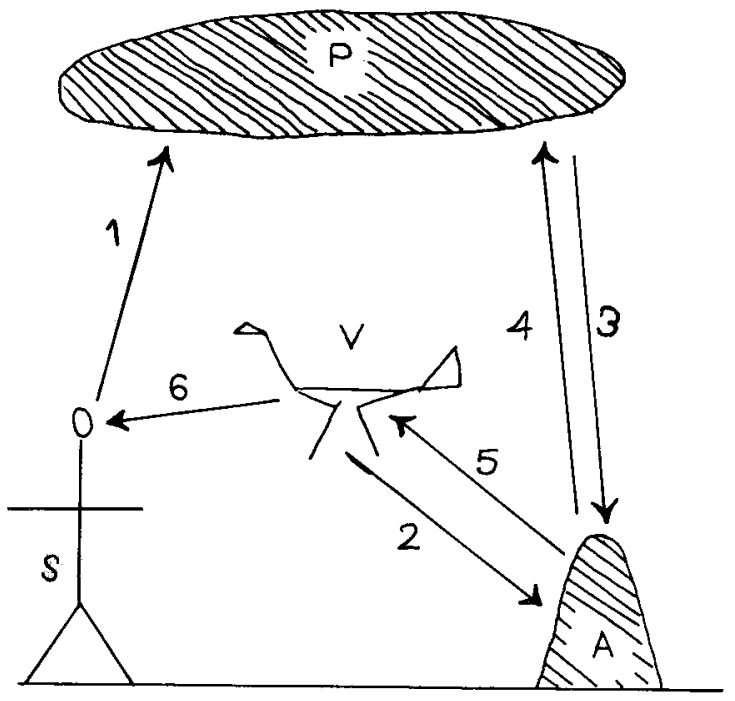

Figure $A$ 
Les phases du sacrifice sont les suivantes :

-- phase 1. Le sacrificateur s'adresse à la puissance pour lui signaler qu'un sacrifice va avoir lieu.

-- phase 2. Il égorge la victime sur I'autel.

-- phase 3. La puissance, alertée par le sacrificateur (phase 1), est appelée sur 1'autel. "L'autel tète le sang de la bête sacrifiêe comme le nourrisson tète sa mère", il nourrit et se nourrit du sacrifice.

-- phase 4. Retour vers la puissance.

-- phase 5. La victime, déposêe sur 1'aute1, se nourrit elle-même de

la force vitale ainsi libérée qui va en particulier dans son foie.

-- phase 6. Le sacrificateur, en mangeant la victime sacrifiêe, prend une partie de cette force vitale.

A la fin de 1 'analyse, Griaule dit :

"Le circuit est ainsi fermé. Il est fait de canaux visibles ou audibles : parole, sang, bol alimentaire (côté humain) et de canaux invisibles : trajet PA, AP, AV (côté surhumain). Dans ce dernier cas, le nyama est à la fois le véhicule et le vêhiculé, le conduit et ce qui est conduit.

Ce circuit, dans son ensemble, intëresse des forces qu'il ne crée point et qu'aucun des relais (A, P, S, V) ne crêe. Le sacrifice n'est pas action créatrice; tous les actes oraux et manuels de ses agents n'ont d'autre efficacité que de conduire vers un but et selon un circuit voulus une vertu préexistant radicalement dans les êtres et matières mêmes qu'ils utilisent ou supposent. Le sacrifice serait sans objet, nonobstant toute science de procédure, s'il en était autrement. (...) Le sacrifice, chez les Dogons, est donc, dans sa partie centrale, un acte technique déterminant un déplacement de nyama et compris comme tel."

Cet article, daté de 1940, est un excellent document de réflexion sur le problème du sacrifice. J'ai choisi pour son application trois éléments du.sacrifice chez les Songhay, illustrês par trois passages de films.

Le premier est tiré d'un de mes premiers films, "Les magiciens de Wanzerbe" ; il s'agit du sacrifice d'un taurillon au gina d'une montagne, un génie de lieu.

Le matin de bonne heure, tous les hommes du village, accompagnés par les jeunes garçons, se rendent à la montagne de sargume. Ils y conduisent un jeune taureau blanc. Les enfants vont ramasser du bois. Puis, l'animal est couché sur le sol, il est égorgé, le sang coule sur une pierre. Les hommes surveillent le dessin du sang : il est favorab1e. L'animal est ensuite dépecé et découpé. Il est grillé sur le feu de bois et mangé sur place. Les restes et la peau sont enterrés à 56 
côté de la pierre du sacrifice. Les jeunes garçons rentrent en courant vers le village. Les hommes partent seuls vers la montagne de sargume. Dans ce sacrifice (figure $B$ ), la puissance $P$ n'apparaît jamais. Elle est sous-entendue, $\mathrm{P}$ et $\mathrm{A}$ sont confondus. Dans ce premier type, nous avons donc le réseau suivant :

-- phase 1. Le sacrificateur s'adresse au gênie du lieu.

-- phase 2. Il abreuve la montagne.

-- phases 3 et 4. Elles n'existent pas, mais sont implicites.

-- phases 5 et 6. Elles existent

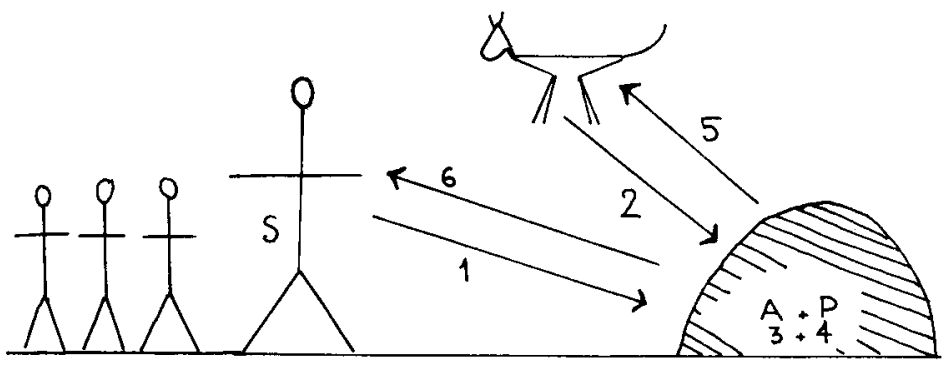

Figure B

Dans le deuxième film, il s'agit du sacrifice annuel du yenendi de 1'ouverture de la saison des pluies, destiné à sceller l'alliance entre les hommes et les gérnies responsables du ciel. Les génies ont parlé avec les hommes et, après avoir donnê aux hommes les raisons pour lesquelles la saison précédente avait été mauvaise, ils se sont mis d'accord avec eux sur la nouvelle saison des pluies. A la fin de la cérémonie, les prêtres sacrifient un bêlier blanc à l'une des divinités magistrales, à ce serpent de toutes les couleurs, sajora, "l'arc en ciel", représentê par un arbre fourchu ; c'est lui qui arrête la pluie avec son dos pour en remplir les puits :grâce à lui, en dehors de la saison des pluies, les hommes ont de l'eau.

Tous les prêtres et les danseurs de possession se groupent autour de l'arbre de l'arc en ciel. Un bélier blanc est renversé aù pied de l'arbre et égorgé. Le prêtre magistral wadi recueille le sang dans une calebasse et en arrose soigneusement la branche de l'arbre de l'arc en ciel.

Le sacrifice (figure C) est différent du précédent : 
-- la puissance $P$ (Ps) est l'ensemble des divinités du ciel responsables de la saison des pluies.

-- les animaux sacrifiés $V$ sont réservés aux prêtres qui les mangent. En effet, ce sont les prêtres qui sont responsables, qui ont organisé la cérémonie, qui ont parlé aux dieux.

-- il y a plusieurs sacrificateurs $S$ (Ss), qui peuvent être des hommes et des femmes.

-- l'autel A est 1'arbre fourchu dressé près de la "case des génies".

-- la victime $V$ est un bélier blanc.

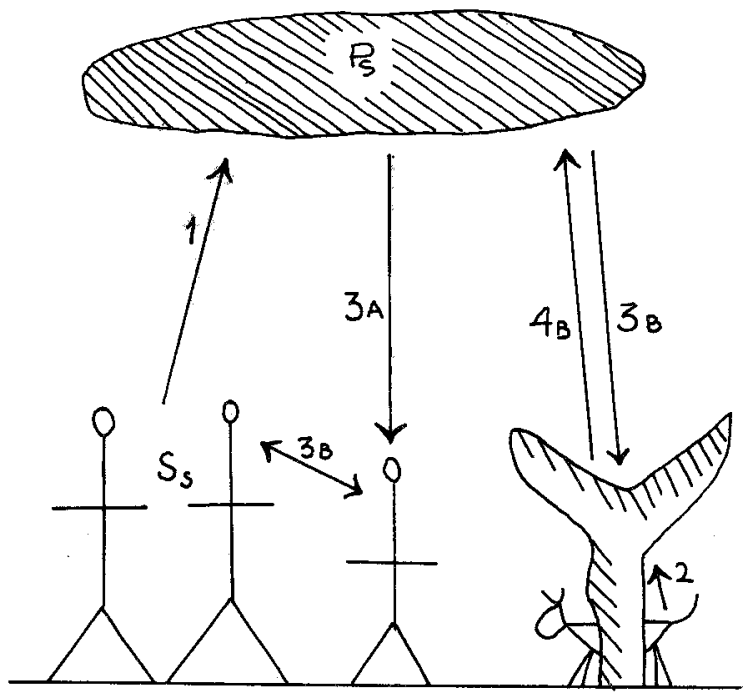

Figure $\mathrm{C}$

Les puissances du ciel sont, venues dans 1 a phase 3 s'incarner dans le corps des gens qui sont possédés, 3A. Il y a eu contact direct, 3B, entre Ps et Ss par l'intermêdiaire de la possession. Les divinités étant réparties en Ps, le sacrifice a lieu suivant le schéma : 3B, 4B ; le 2 va directement sur l'arbre, le 6 revient aux sacrificateurs.

Ainsi, dans ce deuxième type de sacrifice, il y a eu une séquence supplémentaire qui a permis de passer un contrat entre les hommes et les dieux, contrat qui a été. "scellé" par une libération de force vitale. 
Le troisième sacrifice est totalement différent; il va non plus $\mathrm{s}^{\prime}$ adresser d̀ $\mathrm{P}$ mais va être effectué contre P. I1 s'agit de voler une âme à Dieu et de la mettre dans un autel, c'est-à-dire de créer un nouvel autel doué de force vitale, un toom. Pour garder cette âme, on fait un faux enterrement conforme à la religion la plus importante de la rêgion (ici l'islam). Ce faux enterrement est suivi du véritable enterrement au cours duquel on transfert l'âme du mort dans une boite qui deviendra la base d'un autel de famille auquel on devra sacrifier, ou du culte villageois de la montagne où s'est déroulée la cérémonie, ou enfin du culte plus général d'une nouvelle divinité, holey, qui pourra s'incarner sous la forme d'un génie de la danse de possession. Bien entendu, une cérémonie de ce genre est privée ; il est impensable d'y assister, donc de la filmer. Ce film est celui d'un simulacre provoqué mais qui a dépassé les intentions que nous avions (tourné à l'occasion d'un film de fiction, "Babatou, les 3 conseils").

Après un" simulacre d'enterrement islamique, les guerriers suivent un magicien sur une colline boisée. Le cadavre, cousu dans une peau de boeuf et portant ses armes brisẻes, est posê sur le sol. Le magicien s'adresse aux quatre points cardinaux. Il fait sur lui-même un test d'invulnérabilité ; puis il égorge un bélier blanc, sans lui rompre le cou. Il lèche trois fois le couteau ensanglanté, puis prend du sang dans sa main pour le boire (quatre fois), enfin il verse le sang dans une boite de peau (batta), emplie à moitié de lait frais. Il se lève, enjambe l'animal sacrifié immobile et le cadavre, prononce une formule magique et, à reculons, enjambe à nouveau le cadavre et l'animal. Au moment où il enjambe le bélier, celui-ci se met à bouger. Le magicien lui parle et fait emporter le cadavre du guerrier dans un fourré, alors que le bêlier se débat.

Le magicien boit le lait mêlangé au sang dans la boite de peau; puis, après avoir présenté cette boite aux quatre points cardinaux, il la referme et la remet aux guerriers : l'âme du mort s'y trouve enfermée; si on la nourrit de sang, elle deviendra un toom, un autel magique (figure $D$ ).

La phase 6, consommation, a été faite sur place ; le sacrificateur a bu le sang frais du sacrifice, mais nous n'avons été que deux à manger le bélier. Pour les autres, la viande était impure.

Il y a un point sur lequel je n'ai pas d'explication : c'est cette 


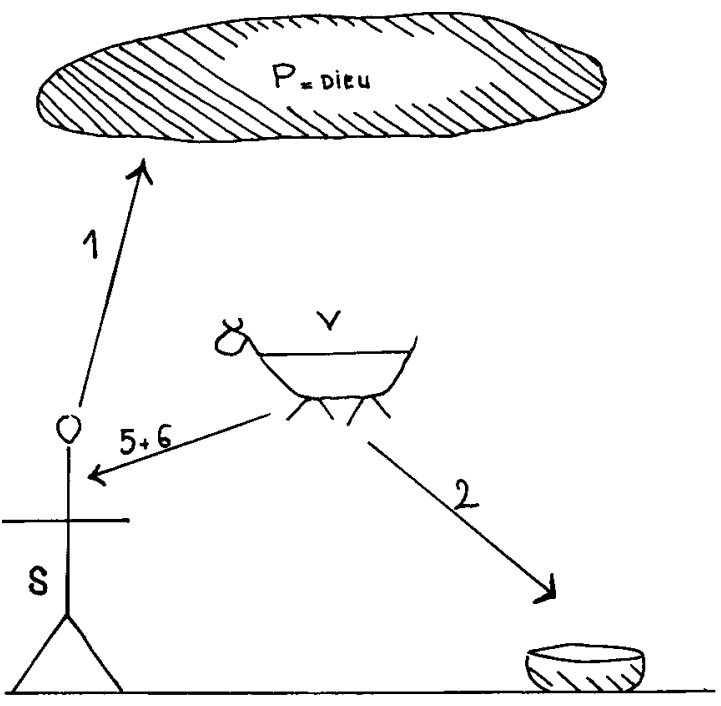

Figure D

Il y a un point sur lequel je n'ai pas d'explication : c'est cette maîtrise de la mort. Comment le sacrificateur peut-il savoir exactement à que1 moment 1 'animal qu'on a égorgé et dont on n'a pas cassé la colonne vertébrale se remettra à bouger ? Car c'est seulement lorsqu'il l'a enjambé une première fois en marche arrière que le mouton s'est mis à souffler ; il s'est débattu lorsqu'il l'a enjambé une seconde fois.

Les textes prononcés sont un charabia mélangeant quatre langues (songhay, tamashek, hausa, arabe), dont une partie a été traduite après la projection du film.

Ce sacrifice est très particulier, car si effectivement il n'est pas réglementaire, il n'est pas pour autant truqué. Que veut dire, par exemple, cette phrase énigmatique : "Les nombreuses morts vont devenir une seule, l'homme ou l'animal cela va devenir une seule mort." (1).

(1) Lorsque j'ai projeté le film au Niger, on m'a donné cette interprétation : le sacrificateur a pensé qu'il s'agissait d'un sacrifice conjuratoire pour la sécheresse et que les morts d'animaux et d'hommes allaient diminuer. Mais peut-être s'agit-il de toute autre chose... 


\section{Sacrifice et transfert des âmes chez les Songhay}

Je n'ai pu faire qu'une esquisse du schéma de ce sacrifice. Le sacrificateur s'adresse à la puissance mais c'est pour lui fermer les yeux. Il a fait la phase 1 , mais il n'y a plus de 3 ni de 4 . On a escamotê un mort avec une fausse tombe qui trompera l'ange de la mort et le vrai cadavre laissé en brousse sera dévoré par les animaux sauvages. I1 n'en restera bientôt aucune trace.

Lorsqu'il s'agit d'un guerrier, les morceaux de son cadavre peuvent servir de charmes positifs ou négatifs, soit contre les camarades du guerrier, soit pour renforcer leur propre pouvoir. Lorsque $j$ 'ai fait un film sur les funérailles du Moogo naaba, le chef des Mossi, on nous a montré le trou dans le mur de sa maison par lequel était sorti le corps au moment même où il y avait dans la cour la tombe dans laquelle en principe $i 1$ avait été enterré. Les indications disaient que le corps enveloppé dans une peau de boeuf était parti faire un périple dans tout le pays mossi. Où allait-t-il ? Quelle serait sa destination? Si je me réfère à Rattray, chez les Ashanti, le chef était promené jusqu'à ce que son corps ne soit plus qu'un squelette dont les os étaient chevillés d'or et placés dans le sanctuaire privé des tombes royales près de Kumasi.

Dans ce troisième sacrifice, la phase l est éliminée ou plus exactement I ne comprend pas de retour. On s'adresse au dieu pour lui fermer les yeux. Le passage essentiel est, pour moi, celui oũ, après avoir prononcé une formule simplifiēe, le sacrificateur s'adresse aux quatre points cardinaux. Il entre alors dans un rituel magique, i1 est au milieu du monde, il est devenu le maître de ce qu'il va faire. L'autel n'existe pas encore, il va crêer un lieu de culte pour nourrir cette âme errante. Il n'y a pas de phases 3 et 4. Dans la phase 2, le sacrificateur utilise simplement un nouveau "véhicule", du lait frais. Il y a donc bien une création. Suivant le schéma de Griaule, on peut dire que l'autel tète le sang. Ici, l'autel commence par boire le lait de sa création. L'autel n'existant pas vraiment encore, le 5 et le 6 sont, pour moi, confondus dans l'ingestion directe par le sacrificateur. Il n'y a pas ici de circuit fermé ; il y a oblitération de la puissance divine; la force vitale active est désormais dans cette boite. Il y a un élément singulier : le sacrificateur a bu le sang en 5 et en 6 . Ce schéma est donc totalement différent des autres. 


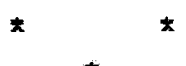

Essayons maintenant d'analyser tous ces schēmas, en tentant de déterminer "l'économie" de la force vitale en circulation.

Le premier schêma (A) est simple : la mort d'un animal a renforcé le nycama de la puissance de 1 'autel et du sacrificateur qui représente la société. Il y a circuit fermé.

Dans le deuxième schéma (B), le circuit est à peu près le même ; la viande doit être consommée sur place par les jeunes garçons qui représentent la communautê ; la montagne est une divinité protectrice des rếcoltes, c'est un ancien lieu de culte gourmantchê -- premiers maîtres du sol -- que les Songhay ont assimilê. L'économie des forces vitales est simple : il y a eu renforcement de la force vitale des hommes du village et de leur montagne protectrice; donc circuit fermé.

Dans le troisième schéma $(C)$, le sacrifice fait à l'arbre de l'arc en ciel scelle l'alliance entre les hommes et les dieux du ciel qui étaient incarnés dans les danseurs de possession; on peut admettre qu'il y a aussi un circuit fermé : le sacrifice renforce la force vitale des divinités du ciel qui donneront la pluie et celle des hommes qui participeront au cycle agraire à venir. Mais on voit apparaître là une notion nouvelle, celle de bya, de "1'âme", qui est absente du schéma de Griaule.

Dans le quatrième schéma (D), cette notion d'âme est évidente. Que s'est-t-il passé ? Ce qu'on a volé à Dieu, ce n'est pas la force vitale du guerrier puisqu'il est déjà mort. C'est un bya qu'on a dérobé à Dieu, une âme. I1 y a création d'un aute1 nouveau qui demandera aux hommes un entretien annuel.

I1 faudrait reprendre tous ces schémas du sacrifice, en y ajoutant le circuit économique des âmes; dans le schêma A, Griaule ne parle pas de l'âme libérée de la victime; or la victime a un "double", une âme ; que devient ce "double" ? En phase 6, quand, après le sacrifice, le sacrificateur, avalant le bol alimentaire, renforce son nyoma, quel est le rapport entre son âme et celle de l'animal sacrifié ? Doit-il se la concilier, ou la chasser ? Griaule n'en dit rien.

Dans le sacrifice à la montagne (schéma B), la situation est identique, il n'est pas question d'âme explicitement, à moins que le génie 62 
de la montagne de Sargumé ne soit effectivement le "double" d'un ancêtre protecteur dont on a transféré l'âme dans cette montagne et auquel on s'adresse en fait. Quand les enfants, nourris de viande, sont rentrés en courant, le travail des forces vitales de la génération à venir est terminé. Mais les hommes sont restés et se sont séparés. C'est alors que se déroule la partie la plus importante de la cérémonie : le contact du "double" des magiciens avec le "double" de l'ancêtre de la montagne.

Dans le sacrifice à l'arc en ciel (schéma $C$ ) le "double" joue un rôle majeur, car le sacrifice ne pourrait avoir lieu si les dieux euxmêmes, par l'intermédiaire de leur "double" ne l'avaient demandê : les "doubles" des dieux incarnés ayant momentanément chassé les "doubles", leurs "chevaux" prennent la parole et exigent le sacrifice.

Dans le quatrième schéma (D), il s'agit d'un vol d'âme. Elle a êté placée dans un réceptacle de force vitale qui lui permettra de continuer à exister (on a rappelé 1 'âme errante d'un corps sans sêpulture). Le corps était le dernier élément qui lui donnait une attache. Cette âme était nulle part; elle ne pouvait aller dans 1'au-delà qu'après l'enterrement de son corps. Elle rôdait. L'appel du sacrificateur aux quatre points cardinaux s'adressait à cette âme errante (qui risquait de devenir un fantôme méchant, buko laalo) qu'il allait contraindre à se fixer dans la boite en libérant à sa place l'âme de l'animal sacrifié. La boite, disposée auprès d'un arbre du lieu, ou dans une case que 1 'on y dressera, deviendra un toom, un autel douê de pouvoir. Les hommes viendront y sacrifier régulièrement comme sur la montagne de sargume (schéma B).

Mais si plus tard les descendants, responsables du culte, abandonnent les sacrifices réguliers, l'âme de l'ancêtre mort redeviendra libre. Elle pourra posséder quelqu'un (un descendant responsable) au cours d'une danse de possession, elle sera un nouveau génie, un nouveau holey; ou bien, elle redeviendra une âme errante, un buko laato, un fantôme méchant dont les hommes devront continuellement se protéger.

G. Dieterlen : Le travail de Griaule a été fait à l'occasion d'un 
événement fortuit : un sacrifice offert par son père avant le mariage d'un de nos informateurs. Ce dernier n'effectuait pas lui-même le sacrifice mais devait y assister pour manger une partie du foie de la victime; car s'il ne consommait pas le foie, le circuit n'était pas ouvert, il n'y aurait pas de dégagement de force vitale et l'intéressé ne bénéficierait de rien. Il s'agit ici d'un sacrifice individuel, réalisé par un père pour son fils au moment de son mariage, qui suit rigoureusement le schéma traditionnel.

$J$. Rouch : C'est donc bien une figure abstraite d'un sacrifice possible.

L. de Heusch : Il serait vain de chercher dans une même société un modèle unique de sacrifice. Si on situe ces différents sacrifices dans leur contexte particulier, le système n'est pas le même. Il semble que le premier sacrifice soit liê à un culte de magiciens chamans, le second au culte de possession. Le troisième enfin implique une rupture de communication. C'est un renversement des modèles : fondation d'un autel avec détournement d'âme. Je pense que pour approfondir ces différences au niveau symbolique, il faudrait examiner l'ensemble des particularitês spécifiques. Le lait apparaît comme l'élément le plus pertinent. Utilise-t-on les mêmes animaux dans ces divers sacrifices?

J. Rouch : Oui, ce sont toujours des animaux à robe, généralement des bêliers ou des taurillons. Pour la dernière opération, il fallait un bêlier blanc.

L. de Heusch : Le lait est-il du lait de vache?

J. Rouch : Oui. Dans le cas de C, s'il y a une grande famine, les gens peuvent prendre un ou deux coqs en $s^{\prime}$ excusant auprès de la divinité.

Y. Cissé : Oũ va exactement le bya du bélier ? Au Mali, la plupart des hommes responsables ont leur répondant dans un bélier. On peut jeter un sort à quelqu'un en touchant à son répondant; 1 'homme peut en mourir.

J. Rouch : Il est important d'étudier le bya de l'âme des animaux sacrifiés. Dans le dernier cas (D), quand le sacrifice est fini, tout est rompu et Dieu va trouver une âme de bélier à la place de celle du guerrier mort. Je n'ai malheureusement pas d'explication... Au Mali, le bélier, qui est le répondant d'un homme, n'est donc jamais sacrifié ?

Y. Cissé : Rarement, en général, il meurt de sa belle mort.

J. Rouch : Le bélier de l'arc en ciel (C) est sacrifié tous les ans mais immédiatement remplacé : on élève spécialement dans l'enclos de la maison des holey un bélier qui sera sacrifié au prochain yenendi.

G. Dieterlen : Il ne peut pas y avoir de déplacement de nyoma s'il n'est pas dirigê par les âmes : le nyama n'est pas indépendant.

J. Rouch : Dans son texte Griaule dit : "(..) allant de la simple libation à 1'immolation d'animaux, plus anciennement d'êtres humains, suivie de consommation." Quand il y avait un sacrifice humain, y avaitil consommation?

G. Dieterzen : Oui, je pense. L'âme libérée retournait à Dieu. Elle n'était pas dangereuse; le sacrifice n'est pas un meurtre. Tout 64 
le symbolisme du troisième sacrifice évoque ce qui revient directement à Dieu chez les Dogon et les Bambara : les quatre points cardinaux, les éléments; il ne s'agit pas de génies, mais de Dieu.

J. Rouch : Pour lutter contre Dieu on prend les armes de Dieu. Le magicien est 1 'anti-dieu. Il se prend pour Dieu dans sa lutte contre Dieu. Il est le Créateur. Ne pourrait-on dire que Griaule s'est trompé et que le 4 est le bya de l'animal sacrifié ? N'y aurait-il pas un 4 bis ? Que devient cette âme?

G. Dieterlen : Elle va vers Dieu.

L. de Heusch : Les Dogon s'intéressent-ils aux âmes des animaux ?

G. Dieterien : Bien sûr. On ne peut tuer un animal sans prendre des risques considérables. Il y a des autels pour cela, ce sont les autels des chasseurs.

Chez les Dogon, il existe un sacrifice annuel fait au pegu. Le pegu est l'autel protecteur d'un village ou d'une région dans lequel on est censé avoir mis le corps d'une victime humaine enterrée verticalement, un clou enfoncé dans le crâne. Le principe spirituel de cette personne est là, il n'est pas lié aux autels familiaux. C'est génëralement un ancêtre, seul et déplacé, auquel on offre des sacrifices. Ces pegu pouvaient également être réalisés avec le corps d'ennemis pris à la guerre ; leur nyama était très agressif et dangereux pour les gens qui attaquaient le village. Il s'agit donc d'un déplacement d'âme et de personne, mais elles ne sont pas retirées à Dieu.

L. de Heusch : Il existe cependant en Afrique des sacrifices dont le but est de séparer les hommes des dieux. Chez les Nuer, par exemple, le sang n'apparaît pas comme le moyen de faire circuler des forces. Le sacrifice d'exorcisme chez les Thonga arrache l'esprit du corps du possédé pour le fixer sur un autel. Ce sacrifice particulier assure une fonction de détournement.

J. Rouch : Vous n'avez pas de chose semblable chez les chasseurs ?

Y. Cissé : Ce n'est pas pareil. Au Mali, il y a un rituel magique qui consiste à capter le double d'un ennemi et à le transférer dans un animal noir qu'on enterre vivant. Chez les Bambara, il n'y a pas de semblable transfert d'âme.

L. de Heusch : Est-ce que ce sacrifice est réservé aux guerriers ?

J. Rouch : Il est réservé à des gens importants qui ont transgressé toute leur vie les règles de Dieu et dont les descenuants demandent à garder 1 'âme.

Ch. Bertaux : La victime peut-elle être un animal non domestique ?

J. Rouch : On m'a toujours répondu non.

Ch. Bertaux : Si la mécanique était liée au nyoma, un animal sauvage ferait aussi bien l'affaire. Il y a un rapport profond entre 1'animal domestique et les instances humaines.

J. Rouch : En dehors de ce sacrifice, ce sont toujours des sacrifices de brousse. Ils se font dans un bois où les animaux sauvages viendront manger le cadavre. Éventuellement, c'est autour de ce lieu que se créera un jour un village quand il sera passé sous sa protection. 
D'autre part, à propos du bélier, c'est la première fois que je vois un sacrifice dans lequel on ne casse pas la colonne vertébrale de la victime. Ceci nous a tous surpris.

L. de Heusch : Le mélange du lait et du sang est extraordinaire. Ces éléments sont le plus souvent antinomiques.

$J$. Rouch : C'était bien sûr un simulacre, mais je pense que le sacrificateur a fait le sacrifice selon les règles. Il était très près d'une transe peut-être chamanistique.

$Y$. Cissé : Pensez-vous vraiment que l'âme du bélier soit restée dans la brousse pour être mangée par les vautours?

J. Rouch : L'âme du guerrier a été transférée dans la boite. Pour le bélier, je ne sais pas. Je pense qu'elle va remplacer celle du guerrier, mais ce n'est qu'une hypothèse.

Ch. Bertaux : Est-ce que la disposition nord-sud du cadavre sert à bloquer la circulation ?

$J$. Rouch : Non, cette orientation est due au fait que ce pays est islamisé et que le mouton est sacrifié suivant le rituel musulman.

Y. Cissé : Pourquoi ce mouton est-il impur?

J. Rouch : Je n'ai pas dit qu'il était impur mais les participants ont tous refusé de le manger, probablement à cause du sang bu. 